\title{
Glottopol
}

Revue de sociolinguistique en ligne

$33 \mid 2020$

Varia - Liberté, égalité, diversité !

\section{Les rectifications orthographiques de 1990 comme révélateurs du rapport des enseignants à l'orthographe}

Catherine Combaz-Champlaine

\author{
(2) OpenEdition \\ Journals \\ Édition électronique \\ URL : https://journals.openedition.org/glottopol/559 \\ DOI : $10.4000 /$ glottopol.559 \\ ISSN : 1769-7425 \\ Éditeur
}

Presses universitaires de Rouen et du Havre

Référence électronique

Catherine Combaz-Champlaine, «Les rectifications orthographiques de 1990 comme révélateurs du rapport des enseignants à l'orthographe », Glottopol [En ligne], 33 | 2020, mis en ligne le 01 janvier 2020, consulté le 02 octobre 2021. URL : http://journals.openedition.org/glottopol/559 ; DOI : https:// doi.org/10.4000/glottopol.559

Glottopol 


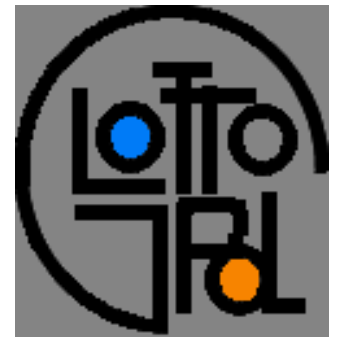

\section{GLOTTOPOL}

Revue de sociolinguistique en ligne $n^{\circ} 33$ - février 2020

Varia : Liberté, égalité, diversité !

\section{SOMMAIRE}

Hommages à Jean-Baptiste Marcellesi : À ringrazià vi, par Niculau Sorba ; “Ciao Ziu ! » par Philippe Blanchet.

Clara Mortamet : Présentation du numéro : Liberté, égalité, diversité !

Manon Him-Aquilli : "Prendre la parole sans prendre le pouvoir ». La mise en registre de l'horizontalité dans les assemblées générales anarchistes/autonomes.

Siham Hocini : La formulation du désaccord dans les débats télévisés algériens : quels "effetsde-politesse "?

Pauline Rannou : Trajectoires de la surdité en France. Parents entendants d'enfants sourds : questionner la fragmentation de l'identité sourde.

Catherine Combaz-Champlaine: Les rectifications orthographiques de 1990 comme révélateurs du rapport des enseignants à l'orthographe.

Marie-Madeleine Bertucci : Éléments d'épistémologie pour l'étude des espaces ségrégués des banlieues urbaines sensibles de la région parisienne.

Élise Gandon: Usages du numérique et illectronisme: nouvelles tâches pour le lecteurscripteur, nouveaux enjeux d'apprentissage.

Marc Arabyan : Quelle langue pour la recherche neurolinguistique française?

José R. de Arellano : Le rapprochement entre l'espagnol et le portugais pendant la décennie dorée sud-américaine : une problématique de l'horizontalité supercentrale.

Adam Wilson: Normes interactionnelles globalisées et communautés de pratique discontinues : les dynamiques sociolinguistiques du tourisme international.

Mélanie Lancien : Caractérisation de la variation liée à la situation de communication : apport de l'acoustique à la phonostylistique.

Assémou Maurice Ludovic Assémou : La prononciation du français parlé en Côte d'Ivoire. 


\title{
LES RECTIFICATIONS ORTHOGRAPHIQUES DE 1990 COMME RÉVÉLATEURS DU RAPPORT DES ENSEIGNANTS À L'ORTHOGRAPHE
}

\author{
Catherine Combaz-Champlaine \\ Université de Cergy-Pontoise, \\ ÉMA (École, Mutations, Apprentissage), ÉA 4507
}

\section{Introduction}

Cet article trouve sa place au sein des différents travaux qui examinent le rapport des enseignants à l'orthographe. Ces nombreux travaux sont orientés par des regards différents. Certains cherchent à comprendre comment les enseignants permettent aux élèves de construire les savoirs orthographiques et proposent des démarches didactiques appropriées (Cogis, 2005 ; Brissaud et Cogis, 2011). D'autres explorent les conceptions de l'orthographe des enseignants du premier ou du second degré à travers leurs témoignages face à la " crise de l'orthographe », leurs «souvenirs d'apprentissage », leurs « réactions face à la faute » et leurs " difficultés » (Millet, Lucci et Billez, 1990). Quant à nous, nous avons cherché à connaitre le positionnement des enseignants du premier degré quant à l'orthographe conçue comme une norme sociale. Cette entreprise est légitimée par J.-M. Klinkenberg qui écrit qu' " il faut remonter au niveau général des normes sociales, telles que les étudie la sociologie, la norme linguistique n'étant que l'une d'entre elles » (2013: 95). Aussi, à travers une analyse des rectifications orthographiques de 1990 réalisée à notre demande par 30 enseignants exerçant dans les classes de CM1 et/ou CM2, nous avons situé leurs positionnements face à ces variations orthographiques licites à l'aune des attributs qui définissent le concept de norme sociale. En ce sens, cette recherche complète les travaux qui interrogent les enseignants sur les rectifications orthographiques (Simon, 2006 ; David, 2011) pour mettre en évidence leur positionnement en tant qu'acteur social dans une société régie par des normes et des valeurs. Nous faisons l'hypothèse que l'orthographe appréhendée comme une norme sociale sert de repère pour juger ces rectifications orthographiques et nuance les adhésions selon les dimensions du concept privilégiées par les témoins.

Dans un premier temps, nous présenterons le cadrage théorique de cette recherche et son objet, à savoir les rectifications orthographiques publiées au journal officiel de la République française le 6 décembre 1990. Puis nous indiquerons la méthodologie utilisée. Enfin nous aborderons les résultats obtenus présentant les positionnements des enseignants face à ces modifications licites. 


\section{Cadrage théorique}

\subsection{Rapport à, logiques d'arrière-plan et norme sociale}

La notion de rapport à analyse les conceptions et les représentations de l'individu à un objet. C. Barré-De Miniac étudie plus particulièrement le rapport à l'écrit (2000). Nous lui emboitons le pas en retenant certains éléments de définition :

Ensemble des significations construites par le scripteur à propos de l'écriture, de son apprentissage et de ses usages. Significations singulières pour les uns, partagées par le groupe social pour d'autres, le groupe culturel pour d'autres encore. L'ensemble étant de toutes manières retravaillé, réorganisé par un sujet unique, ce que désigne le singulier de l'expression rapport à. (Guernier et Barré-De Miniac, 2009 : 204-205).

Notre intention consiste à repérer les significations que prennent les rectifications orthographiques de 1990 chez les enseignants de l'école primaire française, significations d'un groupe professionnel en charge de les enseigner (Programmes d'enseignement du cycle des apprentissages fondamentaux (cycle 2), du cycle de consolidation (cycle 3 ) et du cycle des approfondissements (cycle 4), 2015) et significations singulières pour chaque témoin qui veut - ou pas -les appliquer.

Ce premier cadrage est complété par la notion de logiques d'arrière-plan développée par D. Bucheton (2011). Nous interrogeons l'identité socio-orthographique des enseignants constituée « des valeurs qui renvoient aux différents cadres de l'expérience personnelle, scolaire et sociale, bref, à la culture et à [leur] [...] histoire singulière [...]»(45-46). Nous analysons les positionnements de chacun des 30 témoins en nous appuyant sur la distinction opérée par A. Noyère (2002) qui différencie les postures considérées comme les choix cognitivo-langagiers relatifs à un objet réalisés en situation d'apprentissage/enseignement et les positionnements comme des représentations exprimées dans les propos sur un objet, par les sujets.

Ces positionnements quant aux rectifications orthographiques constituent une facette du rapport à la norme sociale qu'est l'orthographe, considéré comme "la disposition [d'une] personne à l'égard d'un objet » et sa disposition «à l'égard de la mise en œuvre pratique de cet objet dans sa vie personnelle, culturelle, sociale et professionnelle ». (Barré-De Miniac, 2000 : 13)

\subsection{L'orthographe pensée comme une norme sociale}

Le second concept qui délimite notre approche est celui de norme sociale. B. Wynants indique que « [l'orthographe] est un code social tout autant qu'un code linguistique [...] parce que le code lui-même est la cristallisation d'échanges sociaux complexes encastrés et engagés dans la production de la société [...], les pratiques sociales débord[ant] largement du cadre étroit du domaine des pratiques de transcription linguistiques » (1997 : 9-10). Nous regardons donc les rectifications orthographiques comme une norme sociale culturelle, c'est-à-dire comme « les usages communs aux membres d'un groupe, d'un point de vue en quelque sorte "stylistique", à travers les formes sélectionnées » (Demeulenaere, 2003: 250). La norme sociale a un caractère plus ou moins prescriptif et plus ou moins explicite dans la vie sociale. L'auteur en propose la définition suivante :

D'un point de vue sociologique, la norme intervient dans une interaction où des individus exigent le respect, pour eux-mêmes et/ou pour d'autres de certaines conduites d'actions de préférence à d'autres possibles. Cette normativité prescriptive présuppose ainsi la normativité évaluative, mais elle lui ajoute quelque chose de spécifiquement contraignant. Ces normes peuvent ainsi être elles-mêmes justifiées de diverses manières, s'appliquer à des objets divers et correspondre à des exigences de respect [...]. Ces normes 
peuvent être renforcées socialement par des sanctions pratiques, mais cela n'est pas nécessaire: il peut s'agir d'une norme dont le respect s'appuie sur des exigences intellectuelles. (Demeulenaere, $2001: 192$ )

À la suite de P. Demeulenaere, nous situons l'acteur social face à la norme comme n'étant ni entièrement soumis à celle-ci, ni totalement libéré de sa contrainte. C'est pourquoi nous postulons que les enseignants rencontrés n'expriment pas tous le même positionnement face aux rectifications orthographiques de 1990, certains «jouant» avec la norme plus ou moins que d'autres.

Les différents travaux portant sur la norme sociale (Boudon, Demeulenaere et Viale, 2001 ; Demeulenaere, 2003) mettent en évidence cinq dimensions qui la constituent : la norme sociale est régulière, elle est prescriptive, elle est collective, elle est contraignante parce qu'elle impose des sanctions négatives en cas de transgression et elle repose sur des valeurs sous-jacentes qui la justifient.

Ainsi, la norme sociale orthographique s'impose aux individus qui composent avec elle dans les différentes situations de leur vie sociale. Personne ne peut s'en affranchir véritablement. $\mathrm{Ne}$ pas la respecter est stigmatisant. Cependant, différents facteurs rendent cette tâche difficile, tels que la complexité de l'objet culturel qu'est l'orthographe française. C'est pourquoi les rectifications orthographiques de 1990 cherchent à répondre à cette complexité qui peut mettre les usagers en difficulté.

\subsection{L'orthographe et les enseignants}

Il existe de nombreux travaux de recherche traitant de l'orthographe dans le champ de la didactique du français qui en a fait un objet spécifique parce que l'orthographe «a pris une valeur sociale indéniable» et qu'elle est "socialement identifiable comme matière incontournable » (Daunay et Reuter, 2008 : 64). Parmi ces recherches, certaines portent sur les discours des enseignants concernant leurs pratiques professionnelles, d'autres sur leurs représentations de l'orthographe.

Une enquête publiée en 1998 par M.-L. Elalouf, M. Journot, M. Tamine, C. Tisset et R. Tomassone analyse les représentations de l'orthographe de futurs enseignants, lauréats d'un CAPES de Lettres ou du concours de professeur des écoles, à partir d'un questionnaire portant sur trois dimensions : leurs représentations de l'orthographe, leurs représentations de la norme orthographique, leurs représentations de l'enseignement de l'orthographe. Les auteurs mettent en évidence la sous-estimation de la complexité du domaine graphique par les enquêtés, une focalisation de leur réflexion sur les outils pédagogiques aux dépens de la manipulation des aspects scientifiques et de la réflexion didactique et une absence de prise en compte des contraintes psycholinguistiques.

Une autre enquête est publiée dix ans plus tard (2008) par C. Péret, J.-P. Sautot et C. Brissaud portant également sur le rapport à l'orthographe des professeurs des écoles entrant dans le métier relève plusieurs facteurs qui construisent le rapport à la norme orthographique chez les jeunes enseignants : la tradition pédagogique (basée notamment sur les dictées et les exercices du Bled), le poids des souvenirs de leur propre apprentissage orthographique, le discours des collègues expérimentés, l'appréhension du poids de l'orthographe dans la société et l'influence de leur passé universitaire. Ces constats les amènent à confirmer leur affirmation «Dis-moi quel élève tu étais, je te dirai quel prof tu seras » (2006).

J.-P. Sautot (2000, 2002, 2003), qui s'intéresse à l'utilisation de l'orthographe dans la construction du sens en lecture, montre qu'il est nécessaire de prendre en compte le rapport spécifique que les lecteurs ont construit au code orthographique car ils manifestent des types de rapports normatifs différents appelés par l'auteur postures normatives. Selon lui, ces postures normatives sont la résultante de deux composantes : une compétence orthographique et une certaine disposition sociale relative à la norme : 
La posture normative est déterminée par les différents filtres cognitifs que constituent la maitrise des différentes logiques de l'orthographe (phonographie, orthographe lexicale, grammaticale...) d'une part et le rapport à la norme d'autre part. (Sautot, 2008)

Cette disposition sociale envisage la norme orthographique soit comme «une norme de prescription, c'est-à-dire une série d'injonctions positives destinées à réguler les usages, soit comme une norme de proscription destinée à faire respecter les interdits » (2003:4).

Nous interrogeons ici cette "disposition sociale» en regardant comment les maitres se situent par rapport aux attributs du concept de norme sociale quand ils sont confrontés aux rectifications orthographiques de 1990. Il ne s'agit donc pas de repérer un décalage entre leurs discours et leurs pratiques orthographiques réelles (David, 2011) mais de classer leurs arguments à l'aune de ce que représente, pour eux, la norme sociale en les confrontant à 9 formes rectifiées en contraste avec les formes non-rectifiées (Simon, 2006 : 194).

\subsection{Notre objet : les rectifications orthographiques}

Différentes propositions ont été émises pour « réformer »l'orthographe tout au long de son histoire, telles l'écriture phonétique (l'Alfonic, Martinet, 1983) ou les tolérances pour les concours et examens dépendant du ministère de l'Éducation ${ }^{1}(1901,1903,1976)$ en passant par ses différentes fixations dans les dictionnaires de l'Académie. En 1990, des rectifications orthographiques sont publiées au journal officiel. Elles ont pour objectif essentiel d'apporter « des rectifications utiles et des ajustements afin de résoudre, autant qu'il se peut, les problèmes graphiques, d'éliminer les incertitudes ou contradictions, et de permettre aussi une formation correcte aux mots nouveaux que réclament les sciences et les techniques » (J.O., $1990: 3)^{2}$. «Écartant tout projet d'une réforme bouleversante de l'orthographe qui eût altéré le visage familier du français et dérouté tous ses usagers répartis sur la planète », les experts adressent au Conseil supérieur de la langue française des propositions « à la fois fermes et souples : fermes, afin que les rectifications constituent une nouvelle norme et que les enseignants puissent être informés précisément de ce qu'ils auront à enseigner aux nouvelles générations d'élèves ; souples, car il ne peut être évidemment demandé aux générations antérieures de désapprendre ce qu'elles ont appris, et donc l'orthographe actuelle doit rester admise ». Ainsi, certains mots acceptent désormais deux formes orthographiques licites.

Trois grands principes ont présidé à l'élaboration de ce travail :

- la volonté de démocratiser l'orthographe française en apportant des améliorations sur les points qui posent le plus de problèmes, en mettant fin à des hésitations, à des incohérences et à des « scories » de la graphie qui ne servent ni la pensée, ni l'imagination, ni la langue, ni les utilisateurs ;

- l'appui sur les usages qui s'établissent, les tendances à la cohérence déjà repérables et les évolutions déjà amorcées ;

- la volonté de proposer des modifications mesurées qui n'entrainent pas de bouleversements. Ces principes reflètent clairement les attributs du concept de norme sociale :

\footnotetext{
${ }^{1}$ En 1901, Georges Leygues signe un arrêté relatif à la simplification de l'enseignement de la syntaxe française établissant une liste de tolérances aux épreuves et concours dépendant du Ministère de l'Instruction publique. Cet arrêté est abrogé par un arrêté de 1976 signé par René Haby qui établit une liste de tolérances fondées sur l'usage. En 1903, la commission Meyer propose une simplification de l'orthographe refusée par l'Académie sous prétexte de ne pas heurter les habitudes fortement enracinées. En 1905, Ferdinand Brunot et Émile Faguet présentent un rapport officiel au ministre Aristide Briand visant à modifier certains points de l'orthographe, qui sera également écarté.

${ }^{2}$ Rapport présenté par Maurice Druon, secrétaire perpétuel de l'Académie française, président du groupe de travail devant le Conseil supérieur de la langue française, le 19 juin 1990 paru au journal officiel de la République française - Documents administratifs - Les rectifications de l'orthographe, le 6 décembre 1990.
} 
- puisque la norme est contraignante, il ne faut pas que le code orthographique soit une succession de problèmes graphiques ;

- puisque la norme sociale est régulière, le code orthographique doit répondre à des règles ;

- puisqu'une norme ne peut «advenir comme pure nouveauté » (Prairat, 2012: 42), les rectifications orthographiques s'appuient sur des usages déjà présents ;

- puisque toute norme nouvelle se heurte à la stabilité de l'ancienne, les experts ne proposent pas une réforme qui transformerait radicalement l'orthographe française mais des rectifications.

Les enseignants partagent-ils ces considérations?

\section{Méthodologie}

Pour apprécier le positionnement des enseignants de cycle 3 de l'école française à l'orthographe pensée comme une norme sociale, nous leur avons demandé lors d'un entretien semi-directif de commenter neuf rectifications orthographiques.

Nous avons rencontré 30 enseignants exerçant en région parisienne dans des classes de CM1 et/ou CM2. Notre échantillon compte 4 hommes et 26 femmes. Ils ont entre 22 et 56 ans. Leur ancienneté va de 1 an à 35 ans. Leurs études montrent des amplitudes variées : du baccalauréat au DEA ou master 2, ainsi que des contenus de formation divers : 10 d'entre eux ont suivi des études universitaires à dominante scientifique et 16 des études plutôt littéraires ou linguistiques (4 possèdent le seul baccalauréat).

Les 2 phrases qui leur ont été proposées étaient présentées l'une sous l'autre, en même temps et sur le même document, leur lecture pouvant ainsi aller de l'une à l'autre. La phrase était d'abord écrite en orthographe traditionnelle puis elle l'était en orthographe rectifiée. Cet ordre a été adopté pour déstabiliser les enseignants le moins possible car nous postulions que les graphies traditionnelles leur étaient mieux connues. Il nous a paru trop délicat de demander aux enquêtés, lors d'un entretien en face à face, d'écrire sous dictée le texte, car cela aurait pu conduire à stigmatiser leurs hésitations et écarts, ce qui aurait nuit à la qualité et la quantité des échanges ultérieurs. Nous avons pensé qu'il n'était pas facile à un enseignant d'avouer ses difficultés orthographiques et d'en laisser une trace.

Voici ces deux phrases :

« J'amoncelle les pièces dans mon porte-monnaie pour payer le coiffeur qui me frisottera les cheveux un de ces après-midi car cela coûte cent vingt et un euros. » crie de sa voix aiguë la joaillière au visage boursouflé.

«J'amoncèle les pièces dans mon portemonnaie pour payer le coiffeur qui me frisotera les cheveux un de ces après-midis car cela coụte cent_vingt_et-un euros. » crie de sa voix aigüe la joaillère au visage boursoufflé. ${ }^{3}$

Cette phrase a été créée dans le but de présenter en peu de mots neuf types de rectifications différents. Ces graphies rectifiées répondent à certaines des "variations graphiques ${ }^{4}$ " fréquentes observées par V. Lucci et A. Millet, constituant des zones de fragilité (1994).

\footnotetext{
${ }^{3}$ Les rectifications sont soulignées. Elles concernent la conjugaison des verbes en -eler et -eter, l'emploi des traits d'union, les terminaisons des verbes en -otter ou -oter, l'accord pluriel des noms composés d'une préposition et d'un nom comportant de fait un trait d'union, l'emploi de l'accent circonflexe, l'emploi du trait d'union dans le nombre, la présence d'un tréma sur une voyelle et la rectification d'une anomalie en rendant cohérente une série désaccordée.

${ }^{4}$ Le mot variation est employé ici en référence aux travaux sociolinguistiques pour exprimer qu'il existe dans les usages une variabilité graphique de l'orthographe. Les auteurs montrent que les variations produites par les futurs
} 
La consigne adressée aux enseignants était la suivante : «Je vous propose de regarder cette même phrase écrite en orthographe traditionnelle et en orthographe rectifiée. Aucune des deux orthographes n'est fautive. Pouvez-vous commenter ces deux orthographes et notamment l'orthographe rectifiée de la deuxième et me donner votre avis sur celle-ci ? ».

Les propos tenus par chaque enseignant pour chacune de ces neuf rectifications ont été analysés selon les cinq dimensions du concept de norme sociale grâce à une analyse de contenu thématique (Bardin, 2001). Nous avons utilisé ces cinq attributs pour organiser nos opérations de codage et de catégorisation. Le système de catégories étant donné, il s'agissait de « répartir de la meilleure façon possible les éléments au fur et à mesure de leur rencontre. C'est la procédure par 'boites' » (Bardin : 152). La dimension relative aux valeurs a fait l'objet d'une attention particulière qui nous a conduite à la subdiviser en deux sous-catégories correspondant à deux valeurs différentes, le « génie de la langue » (Klinkenberg, 2013) et l'intégration sociale.

\section{Résultats}

Le corpus des arguments des témoins favorables aux rectifications orthographiques ou opposés à celles-ci prend en partie appui sur les attributs du concept de norme sociale (la régularité, l'aspect collectif, la contrainte, la sanction et les valeurs). En ce sens, il révèle leur rapport varié à l'orthographe pensée comme une norme sociale. Les cinq dimensions constitutives de cette dernière ne sont pas toutes évoquées par les témoins ni selon la même intensité. Elles ne sont pas, non plus, toutes proposées par une même personne. De plus, elles ne touchent pas toutes les rectifications. Il n'y a pas un argument unique qui situerait un même type de positionnement pour un même principe de rectification.

Nous dénombrons 98 occurrences évoquant la norme sociale (sur 1350 possibles : 30 enseignants X 9 rectifications X 5 dimensions). Nombre d'analyses portent sur le code linguistique.

In fine, la rectification la mieux acceptée concerne le mot aigüe tandis que celles concernant les mots portemonnaie et coute sont très majoritairement rejetées. Mais au-delà d'une approche quantitative d'adhésion ou de rejet, une analyse qualitative montre en quoi les arguments pour ou contre ces rectifications reposent sur l'identification - ou pas - des attributs du concept de norme sociale.

\subsection{Le caractère collectif}

On dénombre 12 occurrences concernant le caractère collectif de la norme sociale orthographique apposé à ces rectifications. Les arguments des témoins tiennent au constat de la présence ou de l'absence de ces nouvelles graphies dans la société. Celles-ci sont mieux acceptées si elles sont identifiées comme étant déjà dans les usages car elles sont partagées. Lorsque ce n'est pas le cas, les témoins redoutent de faire cavalier seul ou d'être victimes de sanctions injustifiées :

« Si on doit faire des réformes autant utiliser les déformations des mots que les gens utilisent le plus massivement pour un même mot. Donc pour ça, il faut faire des statistiques. De toute manière c'est l'usage par la population qui fait vivre la langue et l'orthographe. On est en démocratie. Pour moi c'est l'usage des gens qui détermine les modifications à opérer. » (femme, 31 ans, 7 ans d'ancienneté, licence d'histoire)

professeurs et les futurs secrétaires interrogés concernent principalement le pluriel des nominaux, les doubles consonnes, les phonogrammes mais aussi les diacritiques tels que l'accent (1994: 82, 93). 
Cependant, les usages sont hiérarchisés et doivent être légitimés. Ils sont admis en fonction du statut de leurs auteurs. Tout le monde ne peut prétendre à réformer l'orthographe. Pour certains enseignants, seuls les écrivains témoignent de cette légitimité : « Il faut que ce soit des écrivains qui ont un usage de la langue mais pas l'homme de la rue qui lui aussi fait usage de l'orthographe parce que l'écrivain il écrit mais il réfléchit aussi et notamment à la forme de la langue » (femme, 37 ans, 8 ans d'ancienneté, licence d'histoire). Pour d'autres, les linguistes ont cette autorité :

« La seule légitimité que je peux accorder à quelqu'un pour réformer l'orthographe, ce serait les linguistes. Pas les académiciens parce qu'ils font partie de l'élite, donc voilà et puis quand on voit qui il y a eu à l'Académie et qui il y a maintenant, on se pose des questions. Pour moi les linguistes font aussi partie de l'élite mais ils étudient la langue parlée telle qu'elle évolue. Ils ne sont pas sur des positions figées d'autrefois comme les académiciens, qui ont une façon d'écrire. La plupart sont des écrivains. Alors que les linguistes sont en connexion avec l'évolution de la langue. Donc si on doit réformer l'orthographe, c'est eux les mieux à même de ne pas la transformer n'importe comment, en regardant les usages. » (homme, 40 ans, 13 ans d'ancienneté, DEA d'astrophysique)

Les enseignants évoquent les problèmes de mise en œuvre des rectifications qui menacent le caractère collectif de la norme sociale. Les usagers de ces rectifications redoutent le fait que peu de personnes les connaissent craignant d'être injustement sanctionnés alors même qu'il s'agit d'une orthographe licite qui ne doit appeler aucune réprobation sociale : «Parfois je n'utilise pas l'orthographe rectifiée parce que je pense que les gens de mon âge vont croire que je fais des fautes d'orthographe et je serais obligée d'expliquer et ça c'est embêtant » (femme, 47 ans, 18 ans d'ancienneté, DEUG langue chinoise).

\subsection{La régularité}

Nous distinguons la régularité de la norme sociale de la régularité des graphies. Si la reconnaissance d'un «moule graphique » (Blanche-Benveniste et Chervel, 1969) est fortement apprécié par les enseignants comme facteur d'unification comme en témoigne leur enthousiasme pour la graphie boursoufflé présentant la double consonne $\mathrm{f}$, la régularité de la norme sociale est toujours évoquée pour s'opposer aux rectifications qui entrainent deux orthographes licites autorisées :

« Moi, je trouve que quand on laisse la possibilité d'orthographier un même mot de deux manières différentes, c'est plus perturbant au final. À mon sens, avoir une seule orthographe, c'est plus sécurisant au final. » (femme, 31 ans, 7 ans d'ancienneté, CM2, licence d'histoire)

Quand deux orthographes sont acceptées, les conditions de leur acceptation sont sérieusement encadrées :

« il faut qu'il y ait une règle qui dise que tel mot peut s'écrire comme ça ou comme ça. Il ne faut pas que chacun se mette à écrire comme il veut. Il faut que ce soit clair et encadré par une règle. En fait il faut qu'il y ait une deuxième norme. On peut avoir été tolérant pour certains mots car ils ont une orthographe difficile mais après il faut s'engager à écrire les mots comme on vient de définir la nouvelle orthographe ou comme l'ancienne orthographe mais pas d'invention personnelle. » (femme, 22 ans, 1 an d'ancienneté, master MEEF)

Les témoins expriment la régularité de la norme sociale orthographique à travers des arguments qui portent sur le caractère logique ou illogique des principes des rectifications proposées : « Pour proposer une réforme il faut qu'il y ait une logique explicative derrière. Moi il faut qu'on me dise il y a une règle, je la comprends et je sais pourquoi, j'obéis. » (femme, 42 ans, 18 ans d'ancienneté, DEA d'économie). Cette règle doit correspondre à la régularité du 
code orthographique pour " qu'on arrête de faire un coup comme ça et un coup autrement» (femme, 27 ans, 2 ans d'ancienneté, licence linguistique-informatique).

De plus, la régularité de la norme sociale est présentée comme un argument en défaveur des rectifications car elle conforte la socialisation orthographique première des enquêtés qui ne veulent pas « changer leurs habitudes » et n'ont « pas envie d'apprendre la nouvelle réforme ». Le regard sur cet attribut de la norme sociale est présent 30 fois dans les entretiens.

\subsection{La contrainte et la sanction}

Les rectifications sont parfois perçues comme des fautes d'orthographe par les témoins bien qu'ils sachent que cette orthographe est autorisée. Les témoins sont attachés à l'orthographe qu'ils connaissent :

«J'amoncèle, au niveau du son c'est le bon, le è fait le même son que les deux $l$. Mais ça change tout de mettre un accent grave parce que le mot il n'est pas le même du tout visuellement. Moi concrètement je ne l'ai jamais vu écrit comme ça avec la nouvelle orthographe, ça me choque. S'il avait fallu trouver les fautes d'orthographe, j'aurais souligné toutes les différences de la phrase 2 et notamment -èle de j'amoncèle. Moi je continuerai à écrire avec les deux $l$. » (femme, 37 ans, 10 ans d'ancienneté, DEA de sociologie)

Une réflexion sur les raisons présidant à ces rectifications proposée lors des entretiens a suscité des positionnements différents. Elle peut n'avoir eu aucun effet déclaré sur la modification d'un comportement personnel "portemonnaie, ça simplifie mais je ne suis pas pour. Moi je reste sur l'ancienne orthographe » (femme, 49 ans, 23 ans d'ancienneté, études d'orthophonie) ou au contraire elle a pu amener un changement d'appréciation de la rectification et une volonté de changement de comportement, la variante n'étant plus jugée comme une transgression ou une faute : « Moi je suis à fond pour la réforme de l'orthographe sur ce genre de chose, pour moi les double-consonnes c'est un enfer, alors oui j'adhère » (femme, 55 ans, 14 ans d'ancienneté, licence d'anglais).

Peu d'avis (3) portent sur cette dimension, les enseignants savent bien que les nouvelles graphies ne peuvent être théoriquement sanctionnées.

\subsection{Les valeurs}

De toutes les dimensions du concept de norme sociale, celle concernant les valeurs constitue le point essentiel à l'aune duquel les rectifications sont examinées et les positionnements justifiés. Elle mobilise 53 interventions.

Une des valeurs qui brille dans la norme sociale orthographique tient à ce que J.-M. Klinkenberg appelle « le génie de la langue » (2013) (13 occurrences repérées). Il se compose de la beauté de la langue, de sa clarté et de son identité. L'autre valeur évoquée est celle de l'intégration dans la société (40 occurrences repérées).

La beauté de la langue ou plus exactement son esthétique idéographique est le plus souvent la valeur de référence pour refuser les rectifications orthographiques jugées laides. Cet argument n'a jamais été présenté pour indiquer une adhésion à la nouvelle graphie : «coûte, c'est joli. Esthétiquement je trouve ça beau l'accent circonflexe. [...] moi je le mets, je ne me vois pas écrire ce mot sans accent circonflexe. » (femme, 49 ans, 23 ans d'ancienneté, études d'orthophonie). Cette position concernant l'accent circonflexe est rappelée par M.-A. Paveau qui cite le traité d'orthographe l'Orthographia Gallica, rédigé à la fin du XIII ${ }^{\text {ème }}$ siècle présentant la beauté de l'accent:

"l's est maintenu pour des raisons esthétiques, l'auteur du traité conseillant de maintenir l's "pur bele ecriture", pour la belle écriture. Voilà donc une des sources de cet attachement passionnel au circonflexe à l'aube du XXI siècle, la transmission d'une 
préoccupation esthétique fixée sur la consonne s [...]. On comprend donc que l's implosif et son héritier le circonflexe président partiellement à la naissance de l'orthographe comme pratique de réglementation de l'écrit, pratique qui n'est pas exempte d'un souci esthétique. 》 (2008: 108)

La clarté de l'orthographe est une autre valeur du génie de la langue sur laquelle repose la norme sociale orthographique. Cette clarté, fondée notamment sur un accès aisé à l'identité visuelle du mot, permet à la pensée du lecteur-scripteur de s'organiser en donnant du sens aux éléments constitutifs du mot et en lui permettant de s'appuyer sur des repères immédiatement disponibles. Les témoins soulignent massivement que les anciennes graphies en sont porteuses alors que les nouvelles graphies la mettent à mal, entravant ainsi la reconnaissance du mot, voire son sens :

«Porte-monnaie en deux mots, ça a du sens. Ça renvoie à l'action et à la dénomination, au nom. Porte ça renvoie à une action, à un verbe particulier qui a du sens, il faut garder les mots composés avec porte en deux mots même portemanteau et portefeuille. Ça m'interpelle parce qu'il y a un vrai sens sur porte, c'est l'action de porter. On a oublié le sens de l'utilité de l'objet. D'ailleurs je ne l'enseigne pas. La nouvelle orthographe, ça me choque.» (femme, 37 ans, 14 ans d'ancienneté, CM2, licence sciences du langage)

Peu d'enseignants pensent que la nouvelle graphie éclaire la pensée : « Des tirets partout dans les nombres ça permet de prendre le nombre comme un tout, en entier alors pourquoi pas. Ça donne une unité au nombre » (femme, 46 ans, 5 ans d'ancienneté, BTS secrétariat). D'autres y voient, au contraire, une perte de clarté :

« En mettant des traits d'union partout ça va empêcher les enfants de bien identifier les classes qui composent le nombre au profit d'un nombre global. Sans les tirets, ça force plus à cloisonner, à marquer la composition en milliards, millions, milliers. C'est plus logique pour faire les classes alors que les tirets qui étirent le nombre. Ça me gêne. Il faudra encore plus réfléchir à ce que ça véhicule au niveau du sens du nombre dans la numération le fait d'en mettre partout alors que moi j'aimais ce cloisonnage qui permettait de bien découper par classe. En fait j'ai l'impression que cette liaison mange les classes. » (femme, 37 ans, 14 ans d'ancienneté, licence sciences du langage)

L'identité de l'orthographe se conçoit à travers la fidélité à un héritage patrimonial que seules les graphies traditionnelles expriment. L'absence d'accent circonflexe dans le mot coute en est l'expression la plus forte, elle cristallise les oppositions avec le plus de virulence :

« coute, le $s$ latin disparait. On n'a plus la référence. Cela me gêne. C'est nier l'histoire du mot. Quand on fait du latin, on voit le mot derrière. L'accent il est là pour nous rappeler à quelle famille appartient ce mot. Je ne l'aime pas comme ça. On perd quelque chose. Je n'aime pas les rectifications parce que j'ai l'impression qu'on dépossède la langue de son histoire, un peu comme si on oubliait son arbre généalogique, la vie commence à toi, alors qu'en fait on est détenteur de l'histoire de sa famille et ça aide à comprendre certains trucs. » (femme, 40 ans, 15 ans d'ancienneté, licence de sciences de l'éducation)

B. Cerquiglini a montré les crispations des français quant à la suppression de cet accent circonflexe que «plus rien ne justifie mais que tout légitime » $(1995: 161)$. Accent du souvenir et témoin de l'histoire de la langue, il est un symbole intouchable de l'orthographe française.

À ces raisons liées au génie de la langue, qui amènent majoritairement les enseignants à rejeter les rectifications orthographiques, s'oppose un argument principal en faveur des rectifications qui tient à l'intégration sociale rendue possible par la simplification technique de l'orthographe. Ce positionnement exprime une valeur sociale de démocratisation qui se désintéresse des aspects liés exclusivement à la langue pour promouvoir l'être humain et social, notamment les élèves, les enfants en général ou les personnes les plus éloignées de la culture 
orthographique $^{5}:$ «L'orthographe ne doit pas empêcher quelqu'un qui ne la maitrise pas d'accéder à une fonction, ne serait-ce qu'une fonction élective, ça ne doit pas empêcher une société d'humains de s'épanouir. Ça ne doit pas être quelque chose qui est tellement déterminant que ça empêcherait quelqu'un qui ne maitriserait pas l'orthographe de trouver sa place dans la société. Alors je suis pour ces simplifications. " (femme, 47 ans, 18 ans d'ancienneté, DEUG langue chinoise) ; «C'est bien pour les enfants ou pour ceux qui n'ont pas compris l'autre orthographe et qui ne la maitrisent pas. Cela simplifie tout et on peut s'intégrer plus facilement dans la société. » (femme, 24 ans, 1 an d'ancienneté, master MEEF).

La prise en considération d'autrui est déterminante dans l'identification et la promotion de cette valeur : "Moi, j'ai une habitude avec l'orthographe classique, mais je dois enseigner les rectifications aux enfants de cette génération. Après, c'est que mon habitude et je ne vais pas imposer mon habitude à ceux qui ne l'ont pas sous prétexte que je veux faire passer mon habitude. » (femme, 50 ans, 24 ans d'ancienneté, DEUG de psychologie).

Se fondant sur la distinction proposée par O. Tschannen (1989) entre intégration sociale (manière dont les individus sont attachés à la société) et cohésion sociale (touchant à l'emprise d'un système de normes sur les acteurs sociaux), B. Wynants constate également que l'accueil favorable aux rectifications orthographiques porte « le souci d'accroitre la capacité de la société à intégrer des individus dans un système social. » (1997:238).

Les rectifications orthographiques sont alors l'expression d'un bénéfice social, d'une forme de démocratisation grâce à une meilleure prise en compte des usagers, permettant ainsi de lutter contre l'élitisme social.

Résistants et partisans des rectifications de 1990 s'opposent sur l'identification des valeurs prioritaires qui sous-tendent la norme sociale. Les premiers privilégient le génie de la langue tandis que les seconds mettent en avant l'intégration sociale. Certains positionnements rencontrés ravivent, 25 ans plus tard, les conclusions d'A. Millet, V. Lucci et J. Billiez indiquant que toute «réforme » de l'orthographe est envisagée selon un paradoxe : on peut changer l'orthographe mais il faut la conserver car il ne faut pas perturber la langue, la culture, la littérature et l'histoire françaises. L'orthographe est un « monument faisant partie du patrimoine national, et devenue langue, elle contribue même à constituer l'État Nation » $(1990: 225)$. D'autres positionnements, au contraire, expriment que cet État-Nation ne doit pas se construire à partir d'un monument mais à partir de ses individus.

Si les avis des enseignants divergent quant à leur rapport à la norme sociale orthographique pour 7 des rectifications proposées, il en est deux qui n'appellent aucun commentaire relevant de la normativité. Il s'agit, tout d'abord, de l'orthographe du mot joaillière/joaillère car aucun enseignant interrogé ne sait le prononcer avec assurance, chacun d'entre eux cherchant alors, exclusivement, à faire correspondre leur prononciation à une des deux propositions graphiques. Il s'agit ensuite du mot boursouflé/ boursoufflé qui est exclusivement examiné à l'aune d'une permanence idéographique, sémantiquement repérable. Ces hésitations montrent qu'une réflexion sur la norme sociale exige d'abord des connaissances linguistiques techniques suffisamment sûres.

\footnotetext{
${ }^{5}$ Nous n'avons retenu ici que les propos qui indiquent explicitement un bénéfice social permis par ces rectifications pour des destinataires variés et précisés : les enfants, les élèves, les adultes, les enseignants, tout le monde... Ces rectifications simplifiant les graphies et permettant ainsi d'orthographier avec plus de facilité et de réussite offrent la possibilité d'échapper à un jugement social sévère quand l'orthographe n'est pas respectée.
} 


\section{Que font les maitres de l'enseignement de ces rectifications en classe ?}

Si l'intérêt assigné à l'enseignement de l'orthographe à l'école, en tant que discipline scolaire appartient au registre des finalités que, D. Hameline définit comme « une affirmation de principe à travers laquelle une société (ou un groupe social) identifie et véhicule ses valeurs » (1979 : 97), les différents programmes officiels $(2007,2008,2015)$ sont muets ou peu loquaces sur ces finalités éducatives et sur les valeurs qui les déterminent. Concernant l'orthographe rectifiée, seule une note technique demande aux maitres de l'enseigner, ce qui ne semble pas suffisant à impulser cet enseignement pourtant jugé, démocratisant ${ }^{6}$. En effet les enseignants s'octroient une certaine liberté qui tient à leurs convictions personnelles et aux types des rectifications orthographiques. Nous distinguons ici trois profils d'enseignants.

Le premier montre un engagement professionnel affirmé à enseigner les rectifications : « $\mathrm{Si}$ tu enseignes le verbe amonceler au présent à tes élèves, tu n'enseignes pas les deux [orthographes]. Moi, je n'enseigne que la révisée » (femme, 50 ans, 24 ans d'ancienneté, DEUG de psychologie). Une seule personne compose ce groupe.

Le deuxième groupe réunit les enseignants qui s'engagent progressivement dans cet enseignement car ils ont pris connaissance des rectifications orthographiques : «coute, mon œil n'est pas choqué parce que j'ai déjà commencé à travailler là-dessus. Mais ça demande encore un raisonnement, comme cela le demande encore pour les enfants quand je le leur enseigne car c'est le tout début » (femme, 42 ans, 18 ans d'ancienneté, DEA d'économie). Ce groupe est également peu nombreux ( 3 personnes).

Le dernier groupe est très majoritaire (26 personnes). Il réunit les enseignants qui n'enseignent qu'occasionnellement les rectifications, soit parce que c'est la seule orthographe dont ils disposent à titre personnel pour certaines graphies ( « moi, de toute manière, j'ai toujours mis un s au pluriel à après-midis », femme, 22 ans, 1 an d'ancienneté, master MEEF), soit parce qu'ils les jugent, pour certaines d'entre elles, tolérables (et d'autres intolérables). Ainsi une enseignante module ses adhésions et déclare successivement : "moi, je reste sur l'ancienne orthographe porte-monnaie, je ne proposerais pas cette orthographe comme possible à mes élèves. Ce n'est pas envisageable », " frisotera, pour les élèves, je pourrais proposer les deux », " après-midi, moi je n'accorde pas mais je ne vais pas le souligner dans une copie d'élève ", " coute, l'accent circonflexe, je le rajoute systématiquement en classe », "cent-vingt-et-un, on s'est mis d'accord pour enseigner les traits d'union aux élèves en conseil des maitres dans l'école », «boursoufflé, je peux ne pas le sanctionner auprès de mes élèves » (femme, 49 ans, 23 ans d'ancienneté, études d'orthophonie).

Ces comportements aléatoires peuvent sembler paradoxaux au regard de la démocratisation scolaire offerte par les rectifications orthographiques, ils indiquent la marge de liberté de l'acteur social.

\section{Conclusion}

Les commentaires des enseignants interrogés sur ces rectifications orthographiques montrent comment ils les réfèrent à la norme sociale pour les juger. Pris entre des positions de fixité dues à leur adhésion au génie de la langue et des positions d'évolution dictées par une volonté de démocratisation, ils les observent à l'aune d'arguments rationnels et collectifs mais aussi d'arguments personnels et affectifs rendant difficile l'expression d'un avis unique et tranché. Chacun exprime à la fois son rapport personnel et professionnel au code et à la norme sociale orthographiques et compose avec cette dernière de manière singulière. Les arguments

\footnotetext{
${ }^{6}$ Rappelons que les travaux de B. et P. Pothier ont montré que les élèves de l'école primaire maitrisent, en général, plus précocement l'orthographe rectifiée que l'orthographe traditionnelle (2016).
} 
favorables aux nouvelles graphies s'expriment lorsque les dimensions de la norme sociale orthographique sont repérées par les témoins dans les rectifications. En revanche, les arguments défavorables à celles-ci montrent qu'elles peuvent être rejetées au motif qu'elles ne constituent pas la norme sociale connue et reconnue.

L'orthographe, bien que pouvant apparaitre à certains comme étant belle, claire et héritée recèle néanmoins de nombreuses difficultés qui « déclassent » toute une partie de la population. Dans cette perspective, les rectifications sont perçues par certains enseignants comme des simplifications démocratisantes, c'est-à-dire comme des améliorations et des adaptations. Pour cette même raison, M. Druon indique qu'elles sont fondées sur le souci d'utilité pour répondre aux difficultés de tous. Il semble que cette idée d'amendement et de perfectionnement repose sur les valeurs de cohésion et d'intégration sociales. Cependant, les rectifications sont aussi analysées en fonction des transformations du code graphique. Les enseignants interrogent et commentent les différences d'encodage en se référant aux principes phonographique et sémiographique de l'orthographe et ce prioritairement en cas de doute, l'urgence étant alors de stabiliser un codage graphique. L'insécurité linguistique des enseignants quant à certaines orthographes proposées les contraint alors à une analyse exclusive du code graphique, comme si, le regard sur la norme sociale ne pouvait advenir qu'après une réassurance graphique pressément recherchée. La nécessité de comprendre l'argument graphique prime et appelle quasi-immédiatement une référence à la socialisation orthographique première des enseignants. S'intéresser à l'analyse de l'orthographe en termes de norme sociale apparait alors comme une méta-analyse.

Cette double approche de l'orthographe comme norme sociale et comme codage graphique donne lieu à une réflexion des enseignants qui s'inscrit dans des espaces - temps personnel et professionnels mêlés.

Notre hypothèse n'est donc qu'en partie validée. Cette étude indique que les enseignants se montrent en même temps, "engagés dans des entreprises modernisatrices » et "résistants au nom de l'authenticité des émotions, des corps et des mémoires. » (Wynants, 1997: 11)

Elle confirme également les connaissances apportées par le groupe RO (Glottopol 19, 2010) obtenues grâce à une enquête internationale qui révèle la variété des réactions face aux rectifications orthographiques de 1738 enseignants et futurs enseignants francophones ${ }^{7}$

En 2016, C. Lelièvre s'interrogeait sur la possibilité pour l'école d'enseigner ce qui reste facultatif dans la société. Cette interrogation résonne d'autant plus fort aujourd'hui que les programmes d'enseignement de l'école primaire revus en juillet 2018 ne font plus explicitement référence à l'orthographe rectifiée rompant avec les directives institutionnelles données et répétées depuis $2007^{8}$. Par ailleurs, la profusion des termes employés ${ }^{9}$ par les enseignants rencontrés pour nommer ces rectifications orthographiques, allant du mot réforme évoquant des changements nombreux et majeurs à celui d'amélioration suggérant un bénéfice qualitatif,

\footnotetext{
${ }^{7}$ Il s'agit d'une recherche menée grâce à des questionnaires auprès de personnes provenant de Belgique, de France, du Québec, de Suisse, d'Algérie et du Maroc, dont les réponses aux questions fermées et ouvertes ont été quantitativement analysées auprès de personnes interrogées provenant de Belgique, de France, du Québec, de Suisse, d'Algérie et du Maroc.

${ }^{8}$ Les programmes du cycle 3 de l'enseignement primaire de 2007, signés par Gilles de Robien, signalent qu' « on s'inscrira dans le cadre de l'orthographe rectifiée. Les rectifications définies par l'Académie française ont été publiées au Journal officiel de la République française le 6 décembre 1990, édition des Documents administratifs. Elles se situent tout à fait dans la continuité du travail entrepris par l'Académie française depuis le XVII ${ }^{\mathrm{e}}$ siècle, dans les huit éditions précédentes de son Dictionnaire. » (BOÉN no 5, 12 avril 2007). Les programmes de 2008, signés par Xavier Darcos indiquent que « l'orthographe révisée est la référence ». Les programmes de 2015, signés par Najat Vallaud-Belkassem stipulent «l'enseignement de l'orthographe a pour référence les rectifications orthographiques publiées par le Journal officiel de la République française le 6 décembre 1990 ».

${ }^{9}$ On trouve ainsi les désignations suivantes : rectification, modification, changement, réforme, amélioration, ajustement, aménagement, simplification, tolérance, transformation, révision, adaptation et correction.
} 
témoigne de différentes représentations de l'évolution du code orthographique qui n'augurent pas toutes d'un engagement quotidien à les mettre en application dans les classes.

\section{Bibliographie}

Bardin Laurence, 2001, L'analyse de contenu, dixième édition, Paris : PUF.

Barré-De Miniac Christine, 2000, Le rapport à l'écriture. Aspects théoriques et didactiques. Villeneuve d'Ascq : Presses universitaires du Septentrion.

Blanche-Benveniste Claire et Chervel André, 1969, L'orthographe. Paris : Maspéro.

Boudon Raymond, Demeulenaere Pierre et Viale Riccardo, 2001, L'explication des normes sociales. Paris : PUF.

Brissaud Catherine et Cogis Danièle, 2011, Comment enseigner l'orthographe aujourd'hui ? Paris : Hatier.

Bucheton Dominique, 2011, L'agir enseignant : des gestes professionnels ajustés, deuxième édition. Paris : Octares Éditions.

Cerquiglini Bernard, 1995, L'Accent du souvenir. Paris : Minuit.

Cogis Danièle, 2005, Pour enseigner et apprendre l'orthographe. Paris : Delagrave.

Daunay Bernard et Reuter Yves, 2008, La didactique du français : questions d'enjeux et de méthodes. Pratiques [En ligne], 137-138, mis en ligne le 15 juin 2008, consulté le 19 décembre 2014. URL : http://pratiques.revues.org/1152

David Jacques, 2011, «Les rectifications de l'orthographe et la formation des enseignants », Le français aujourd 'hui, $\mathrm{n}^{\circ} \mathrm{HS} 01, \mathrm{pp} .245-250$.

Demeulenaere Pierre, 2001, «Normativité et rationalité dans l'analyse sociologique de l'action ", dans R. Boudon, P. Demeulenaere et R. Viale, L'explication des normes sociales, Paris : PUF, pp. 187-202.

Demeulenaere Pierre, 2003, Les normes sociales : Entre accords et désaccords. Paris : PUF.

Dister Anne et Moreau Marie-Louise, 2012, Réforme de l'orthographe française - Craintes, attentes et réactions des citoyens, Glottopol, Revue de sociolinguistique en ligne, $\mathrm{n}^{\circ} 19$, URL : http://glottopol.univ-rouen.fr/numero 19.html

Elalouf Marie-Laure, Journot Michel, Tamine Michel, Tisset Carole, Tomassone Roberte, 1998, «Les futurs enseignants et l'orthographe : représentations et formation », Le français aujourd'hui, $\mathrm{n}^{\circ} 122$, pp. 5-14.

Guernier Marie-Cécile et Barré-De Miniac Christine, 2009, Rapport à l'écrit et construction de connaissances disciplinaires. Étude de cas. Pratiques [En ligne], 143-144, mis en ligne le 13 juin 2014, consulté le 22 décembre 2014. Repéré à http://pratiques.revues.org/1457

Hameline, Daniel, 1979, Les objectifs pédagogiques en formation initiale, en formation continue. Paris : Entreprise moderne d'édition.

Journal officiel de la république française des éditions des documents administratifs : les rectifications de l'orthographe. Repéré à http://www.academiefrancaise.fr/sites/academie-francaise.fr/files/rectifications 1990.pdf

Klinkenberg Jean-Marie, 2013, «L'hydre de la réforme - Images sociales de l'orthographe et de la politique linguistique », dans S. Baddeley, F. Jejcic et C. Martinez (dir.), L'orthographe en quatre temps (p. 73-103). Paris : Champion.

Lelièvre Claude, 2016, «En France, l'orthographe sert à se distinguer socialement », Le Monde du 16/02/2016. Repéré à http://www.lemonde.fr/education/article/2016/02/16/enfrance-1-orthographe-sert-a-se-distinguersocialement 4866346 1473685.html\#RPuKd16Jirq53Veg.99 
Lucci Vincent et Millet Agnès, 1994, L'orthographe de tous les jours. Enquête sur les pratiques orthographiques des français. Paris : Champion.

Martinet André, Villard Jeanne et Martinet Jeanne, Boyer Denise, Dominici Albert et Dominici Gilberte, 1983, Vers l'écrit avec l'alfonic: écoles maternelles et cours préparatoire (Pédagogie pratique à l'école). Paris : Hachette.

Millet Agnès, Lucci Vincent et Billiez Jacqueline, 1990, Orthographe, mon amour. Grenoble : PUG.

Noyère Arielle, 2002, « Genres scolaires et cadres disciplinaires : quels rapports à l'écriture ? », Pratiques, $\mathrm{n}^{\circ} 113 / 114$, pp. 225-242.

Paveau Marie-Anne, 2008, "Chronique linguistique ». Les accents : fonction linguistique ou passion orthographique ? », Le français aujourd'hui, n¹60, pp. 103-110.

Péret Claudie, Sautot Jean-Pierre et Brissaud Catherine, 2008, «Les professeurs des écoles entrant dans le métier et la norme orthographique, Nouvelles recherches en orthographe, Limoges : Lambert-Lucas, pp. 203-214.

Prairat Erick, 2012, "Considérations sur l'idée de norme », Les sciences de l'éducation pour l'ère nouvelle, $\mathrm{n}^{\circ} 45$ (1-2), pp. 33-50.

Pothier Beatrice et Pothier Philippe, 2016, L'orthographe rectifiée à l'école. Paris : Retz.

Programmes d'enseignement du cycle des apprentissages fondamentaux (cycle 2), cycle de consolidation (cycle 3) et cycle des approfondissements (cycle 4) : modification, arrêté du 17-7-2018 - J.O. du 21-7-2018 (MENE1820169A).

Sautot Jean-Pierre, 2000, « Utilisation de l'orthographe et d'autres indices dans la construction du sens en lecture ", Thèse de doctorat de Sciences du langage, Lucci V. (dir), Université Stendhal Grenoble III, 2000.

Sautot Jean-Pierre, 2002, "Orthographe : Construction de quelques "parasites" normatifs en classe, Pratiques de lecture et d'écriture : Des usages sociaux aux savoirs scolaires », Lidil, $\mathrm{n}^{\circ} 25$, pp. 57-70.

Sautot Jean-Pierre, 2002, « Acquisition de postures normatives en rapport avec l'orthographe : Discours et attitudes de l'enseignant dans sa classe ». Communication affichée, Colloque INRP : L'écriture et son apprentissage, Questions pour la didactique, apports de la didactique, Paris 21-23 mars 2002. inrp_final2.pdf

Sautot Jean-Pierre, 2003, "Construction de la norme orthographique: Quelques avatars pédagogiques », Dossiers des sciences de l'éducation, n9, pp. 109-119.

Sautot Jean-Pierre, 2003, « Acquisition de postures normatives en rapport avec l'orthographe : discours et attitudes de l'enseignant dans sa classe », Repères, n ${ }^{\circ}$ 26-27, pp. 103-112.

Sautot Jean-Pierre, 2008, " Le rapport à l'orthographe, qu'est-ce que c'est ? ", Lire au Lycée Professionnel, $\mathrm{n}^{\circ} 60$. SCEREN : CRDP de Grenoble.

Simon Jean-Pascal, 2006, « Perceptions des rectifications orthographiques », dans R. HonvaultDucrocq, L'orthographe en question. Mont-Saint-Aignan : Publications des Universités de Rouen et du Havre.

Tschannen Olivier, 1989, « Anomie et intégration sociale. Fenn, Luhmann et le paradigme néodurkheimien ». Cahiers V. Pareto, n॰83, pp. 123-146.

Wynants Bernadette, 1997, L'orthographe, une norme sociale. Paris : Mardaga. 


\section{GLOTTOPOL}

Revue de sociolinguistique en ligne

Comité de rédaction : Michaël Abecassis, Salih Akin, Sophie Babault, Claude Caitucoli, Véronique Castellotti, Régine Delamotte, Robert Fournier, Stéphanie Galligani, Emmanuelle Huver, Normand Labrie, Foued Laroussi, Benoit Leblanc, Fabienne Leconte, Gudrun Ledegen, Danièle Moore, Clara Mortamet, Alioune Ndao, Isabelle Pierozak, Gisèle Prignitz.

Rédactrice en chef : Clara Mortamet.

Comité scientifique : Claudine Bavoux, Michel Beniamino, Jacqueline Billiez, Philippe Blanchet, Pierre Bouchard, Ahmed Boukous, Pierre Dumont, Jean-Michel Eloy, Françoise Gadet, Monica Heller, Caroline Juilliard, Jean-Marie Klinkenberg, Jean Le Du, Marinette Matthey, Jacques Maurais, Marie-Louise Moreau, Robert Nicolaï, Didier de Robillard, Paul Siblot, Claude Truchot, Daniel Véronique.

\section{Comité de lecture pour ce numéro :}

Mickael Abecassis, Michelle Auzanneau, Salih Akin, Céline Amourette, Gabriel Bergounioux, Philippe Boula de Mareuil, Catherine Brissaud, Aude Bretegnier, Maria Candea, Véronique Castellotti, Régine Delamotte, Jean-François De Pietro, Catherine Delarue-Breton, Anne Dister, Alexandre Duchêne, Valentin Feussi, Françoise Gadet, Stéphanie Galligani, François Gaudin, Monica Heller, Emmanuelle Huver, Caroline Juilliard, Abou Bakri Kebe, Jean-Marie Klinkenberg, Christian Lagarde, Foued Laroussi, Fabienne Leconte, Evelyne Lloze, Nolwenn Lorenzi, Gudrun Ledegen, Nadja Maillard, Marinette Matthey, Bruno Maurer, Véronique Miguel Addisu, Saskia Mugnier, Claire Oger, Marielle Rispail, Richard Sabria, Véronique Traverso, Christel Troncy, Cécile Van den Avenne. 